\title{
The Impact of Emerging 5G Technology on U.S. Weather Prediction
}

\author{
Sarah E. Benish ${ }^{1,2^{*}}$, Graham H. Reid ${ }^{1,3}$, Abhinav \\ Deshpande ${ }^{1,3}$, Shantam Ravan ${ }^{1,3}$, Rachel Lamb ${ }^{1,4 *}$
}

${ }^{1}$ Graduate Science Policy at the University of Maryland (GSP at UMD)

2University of Maryland, Department of Atmospheric and Oceanic Science, College Park, MD

${ }^{3}$ University of Maryland, Department of Physics, College Park, MD

${ }^{4}$ University of Maryland, Department of Geographical Sciences, College Park, MD

*Indicates equal contributions

http://doi.org/10.38126/JSPG170203

Corresponding author: sebenish@umd.edu

Keywords: weather; 5G; forecasting; prediction; satellites; remote sensing; radio bands

\begin{abstract}
Executive Summary: Fifth generation (5G) wireless networks promise to provide faster and more expansive data connectivity, exceeding thresholds from previous fourth generation (4G) technology. The deployment of $5 \mathrm{G}$ infrastructure requires allocating additional frequencies in radio bands at 24 gigahertz $(\mathrm{GHz})$, potentially contaminating neighboring remote sensing bands used for weather forecasting and prediction. The current U.S. out-of-band emissions limit at $24 \mathrm{GHz}$ of $-20 \mathrm{dBW}$ per $200 \mathrm{MHz}$ is projected to degrade meteorological forecast accuracy by up to $30 \%$, reducing the hurricane forecast lead time by 2 to 3 days, and endangering thousands of additional lives. Under the Weather Research and Forecasting Innovation Act of 2017 (Pub.L 115-25), the National Oceanic and Atmospheric Administration (NOAA) must develop more accurate and timely severe weather forecasts in order to protect life and property and reduce economic risk; however, the potential out-of-band interference from the roll out of $5 \mathrm{G}$ threatens this aim. Given U.S. economic reliance on accurate weather prediction (estimated to be in the trillions of dollars), we propose that Congress mandate stricter noise restrictions to adequately meet requirements of the Pub.L 115-25, while minimizing disruption to $5 \mathrm{G}$ deployment.
\end{abstract}

\section{Statement of the issue}

Weather forecasting accuracy has significantly improved over the past 40 years. For example, a modern 5-day forecast is as accurate as a 1-day forecast was in 1980, and useful forecasts now can project 9 to 10 days into the future (Bauer et al. 2015). To achieve this accuracy, weather prediction models require satellite measurements of microwave signals radiated by atmospheric water vapor in the 23.6-24.0 GHz passive band (see Figure 1). While little is known about the out-of-band emissions from developing $5 \mathrm{G}$ technology at $24.24-27.5 \mathrm{GHz}$, there is significant concern that current Federal Communications Commission (FCC) limits on out-ofband noise from 5G are insufficient to ensure accurate water vapor measurements in the 23.6-24.0
$\mathrm{GHz}$ passive band. In particular, the faint water vapor signal at $23.8 \mathrm{GHz}$ is likely masked by $5 \mathrm{G}$ noise, making it nearly impossible for scientists to discern the natural water vapor signal from $5 \mathrm{G}$ interference. Likewise, the $37.0-43.5 \mathrm{GHz} 5 \mathrm{G}$ band could impact data collected in the $36-37 \mathrm{GHz}$ range, which is used for liquid water path and cloud detection (ECMWF 2018).

According to both the National Aeronautics and Space Administration (NASA) and the leading European weather prediction institute, microwave observations provide the largest positive forecasting impact of satellite-based data (Bormann, Lawrence, and Farnan 2019). Microwave observations are estimated to improve short term forecasts by $30-$ 
$40 \%$, the loss of which would reduce forecast accuracy by 3-6 hours compared to current forecasts (ECMWF 2018). Because current technology cannot filter noise from $5 \mathrm{G}$ operations or remove it through post-processing while maintaining current levels of data precision (Lubar et al. 2020), pollution of these bands would significantly degrade data and potentially reduce the reliability of forecasts.

To illustrate the enhancement in hurricane prediction afforded by microwave sensing, consider the microwave image of Hurricane Lorenzo from September 282019 in Figure 2. The image from the Advanced Microwave Scanning Radiometer 2 (AMSR2) instrument utilizes parts of the spectra proposed for 5G. While infrared and visible imagery provide useful information on the structure, intensity, and temperature of a storm (NOAA NESDIS 2019), these tools cannot be used when there is cloud cover. Microwave sensors are particularly useful because of the ability to penetrate the atmosphere, allowing a view inside the hurricane when it is obscured by clouds. This microwave data allows for a more accurate characterization of the intensity and location of precipitation as well as an exact location of the eye, which together provide valuable information about whether the hurricane is intensifying, and thus can enhance our ability to track and forecast the hurricane's future evolution (Lubar et al. 2020). In particular, AMSR2 images from Hurricane Lorenzo were critical to understanding the differences in wind patterns at different levels of the atmosphere, which were not possible with other instruments (JPSS SDOC 2019).

The FCC has proposed an out-of-band emission limit for the $24.25-24.75 \mathrm{GHz}$ band of $-20 \mathrm{dBW} / 200 \mathrm{MHz}$, while the International Telecommunication Union World Radiocommunication Conference agreed to a more stringent level of $-33 \mathrm{dBW} / 200 \mathrm{MHz}$. Neil Jacobs, the acting head of NOAA, testified before the House Committee on Science, Space, and Technology that the proposed FCC limit is predicted to cause a $77 \%$ loss of passive microwave data (US Congress 2019). The study concluded that a stricter out-ofband emission restriction between -45.2 and -52.4 $\mathrm{dBW} / 200 \mathrm{MHz}$ would maintain the integrity of satellite data in the $23.6-24 \mathrm{GHz}$ passive bands (NASA and NOAA 2020).
The periodic nature of satellite orbits may allow $5 \mathrm{G}$ systems to temporarily switch to a different frequency, called time-division multiple access (commonly referred to as "time sharing") (Miao et al. 2016). While already in use by cellular carriers (Lubar et al. 2020), time sharing could affect the accuracy of weather predictions and warnings by causing gaps in NOAA's satellite coverage. Existing proposals to purchase commercial weather data may potentially fill identified data gaps, but there is some concern that tying accurate national weather prediction to non-governmental sources presents risks to national security (Freedman 2019; White House OMB 2020).

\section{Regulatory and Economic Considerations}

The regulatory responsibility of radio bands in the United States is divided between the National Telecommunications and Information Administration (NTIA), which administers federal band use, and the FCC, which oversees private and commercial use (FCC 2020). The FCC has the authority to reallocate bands from government to commercial users through the Commercial Spectrum Enhancement Act (Pub. L. 108-494). Given current White House priorities, (White House 2018; White House 2020), the FCC's strategy to "Facilitate America's Superiority in 5G Technology" (the 5G FAST Plan) is pushing even more bands into the $5 \mathrm{G}$ marketplace, raising $\$ 2$ billion in revenue from leasing shared by T-Mobile and AT\&T after auctioning began in May 2019 (FCC 2016). Despite these efforts, portions of the $5 \mathrm{G}$ roll out directly conflict with the 2017 Weather Research and Forecasting Innovation Act, which mandates prioritizing the development of more accurate and timely warnings of high impact weather events that endanger life and property. Furthermore, the National Integrated Drought Information System Reauthorization Act of 2018 instructs NOAA to establish the Earth Prediction Innovation Centre (EPIC) to accelerate improvements in weather prediction (Pub.L. 115-423).

The rollout of $5 \mathrm{G}$ is expected to enable significant improvements in economic and social goals. The World Economic Forum presents five key drivers of 5G technology: improvements in mobility, reliability, security, band efficiency and power efficiency (World Economic Forum 2020). The successful deployment of these five drivers is estimated to create $\$ 13.2$ 
trillion in global economic value by 2035 , generating 22.3 million jobs in the $5 \mathrm{G}$ global value chain alone (Campbell et al. 2019), but will require vast new infrastructure costing at least $\$ 2.7$ trillion by the end of 2020 (Greensill 2019).

Even with the large economic benefits of $5 \mathrm{G}$ deployment, the importance of passive remote sensing on the U.S. economy, particularly for predicting extreme weather events, should not be overlooked. The average estimated cost of $\$ 1$ billion for severe weather events between 2008-2015 doubled over the past 35 years, increasing global demand for accurate and timely weather services (NOAA NCEI 2020). In the U.S. alone, it is estimated that as much as $30 \%$ of the economy, or $\$ 1.3$ trillion using 2011-2014 growth domestic product (GDP) estimates, is impacted by weather (Dutton 2002), with $3-6 \%$ of the variability in U.S. GDP attributable to weather (Lazo et al. 2011). Revenue from the U.S. forecasting service itself is estimated to be $\$ 500$ million, with the total value of weather data captured across all industries to be in the range of $\$ 13$ billion (NWS 2017).

Acting NOAA Administrator Neil Jacobs testified to Congress that the FCC auction has the potential to degrade meteorological forecasting ability by $\sim 30 \%$ and decrease the hurricane forecast lead time by 2 to 3 days (US Congress 2019), potentially resulting in significant loss of life and property, which NOAA is mandated to protect. Other international research institutions have tested the impact of excluding microwave water vapor data from forecasts, finding that their models would have inaccurately predicted the path of 2012 Superstorm Sandy (McNally, Bonavita, and Thépaut 2014). As the deadliest windstorm in over 40 years, the total economic losses from Superstorm Sandy is estimated to exceed $\$ 70$ billion, making it the second costliest storm in U.S. history after Hurricane Katrina (Allianz Global Corporate \& Speciality 2013).

\section{Policy options}

\section{i. Policy option A: Do not prohibit 5 G Plan from moving forward, but require report}

Let the FCC continue implementation of the 5G FAST Plan and associated band auctioning, without interruption. Within three years of band auctions, require a joint report by relevant federal agencies to evaluate the effects of $5 \mathrm{G}$ use on weather forecasting.

\section{Advantages}

- Likely to gain support from industry given existing financial and technological investments.

- Provides future protection of other bands as necessary based on further scientific assessment.

- Multi-agency reporting increases the likelihood of acceptable recommendations moving forward.

\section{Disadvantages}

- Wait-and-see approach may result in inaccurate prediction of extreme weather events.

- Joint agency research and reporting can be costly and time intensive.

- Proposed recommendations for noise mitigation may apply to $5 \mathrm{G}$ bands not yet auctioned, leaving interference from the 24 GHz unaddressed.

ii. Policy option B: Create new limits on frequency window allocation

Pass new legislation mandating that the out-of-band noise stay below $-50 \mathrm{dBW}$ in the $23.6-24.0 \mathrm{GHz}$ band (superseding current FCC guidelines of $-20 \mathrm{dBW}$ ). Encourage future research on maximum noise levels in other bands with potential effects on weather forecasting performance.

\section{Advantages}

- Proposed limit is strict enough to reduce unwanted interference on weather predictions to near zero.

- Does not prevent the commercial adoption and widespread deployment of 5G.

- Noise level limits may spur innovation in the area of telecommunications; specifically, engineering solutions to maintain frequency integrity, such as changes to modulation schemes, that could have broader use and position the U.S. as a world leader in technical innovation. 
- Research on potential interference on other bands would inform future allocation of frequency bands with respect to current weather prediction capabilities.

\section{Disadvantages}

- Low-noise 5G technology would have to be developed for the already-auctioned $24 \mathrm{GHz}$ band, potentially delaying progress and increasing costs.

- Proposed limits could reduce the usefulness of the $24 \mathrm{GHz}$ band for $5 \mathrm{G}$ and would require new engineering to reduce out-of-band emissions.

iii. Policy option C: Require frequency window time sharing between federal agencies and commercial entities

Mandate time sharing between federal agencies and commercial entities of frequencies from $24.24 \mathrm{GHz}$ to $27.5 \mathrm{GHz}$ and $36.0 \mathrm{GHz}$ to $40.5 \mathrm{GHz}$. Specifically, amend Title 47 CFR $\S 25.259$ to expand the range of frequencies protected for NOAA satellites. Such an amendment could be proposed within H.R. 5000 SHARE Act, which was introduced in the House of Representatives in November 2019. Additional commercial data may be purchased to supplement potential data loss via timesharing.

\section{Advantages}

- The deployment and roll-out of 5G as planned avoids the need for reduced out-of-band noise emissions, which could delay the deployment of $5 \mathrm{G}$.

- No known adverse effects on the accuracy of weather data.
- Reduced costs since time sharing protocols already exist in industry.

\section{Disadvantages}

- Presents risks to national security due to reliance on commercial data to fill gaps.

- Only protects bands from satellites operated by U.S. federal entities.

- $5 \mathrm{G}$ applications that rely on constant high-speed link between two parties might suffer performance loss.

\section{Policy recommendation}

We recommend that Congress implement option B, creating new limits on frequency window allocation. This recommendation has the advantage of allowing the $5 \mathrm{G}$ rollout to continue while still limiting out-ofband emission interference with water vapor measurements critical for accurate weather forecasting. While the direct economic costs of emissions interference are not known, the indirect costs of interference for economies dependent on accurate weather prediction are estimated to be in the trillions of dollars, especially given the increase in unpredictable and extreme weather events due to climate change. Stricter noise restrictions are appropriately precautionary given the enormous risk of adverse impacts. While there are certainly some potential impacts on $5 \mathrm{G}$ planning for commercial entities, this option does not prevent or unduly delay $5 \mathrm{G}$ implementation. Further, it provides an avenue for future band allocation through continued research. Congress should write legislation to protect accurate weather prediction services to adequately meet requirements of Pub.L 115-25, while ensuring $5 \mathrm{G}$ deployment is not critically hindered.

\section{Appendix}

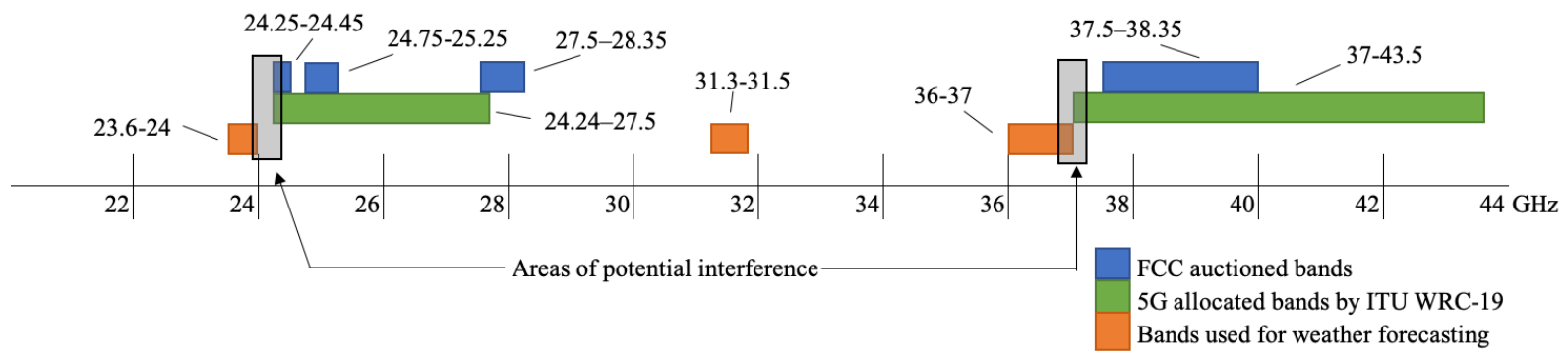


Figure 1. 5G band allocations of the FCC and 2019 International Telecommunication Union (ITU) World Radiocommunication Conference (WRC-19) compared to existing spectra used for weather prediction. Image credit: Shantam Ravan.

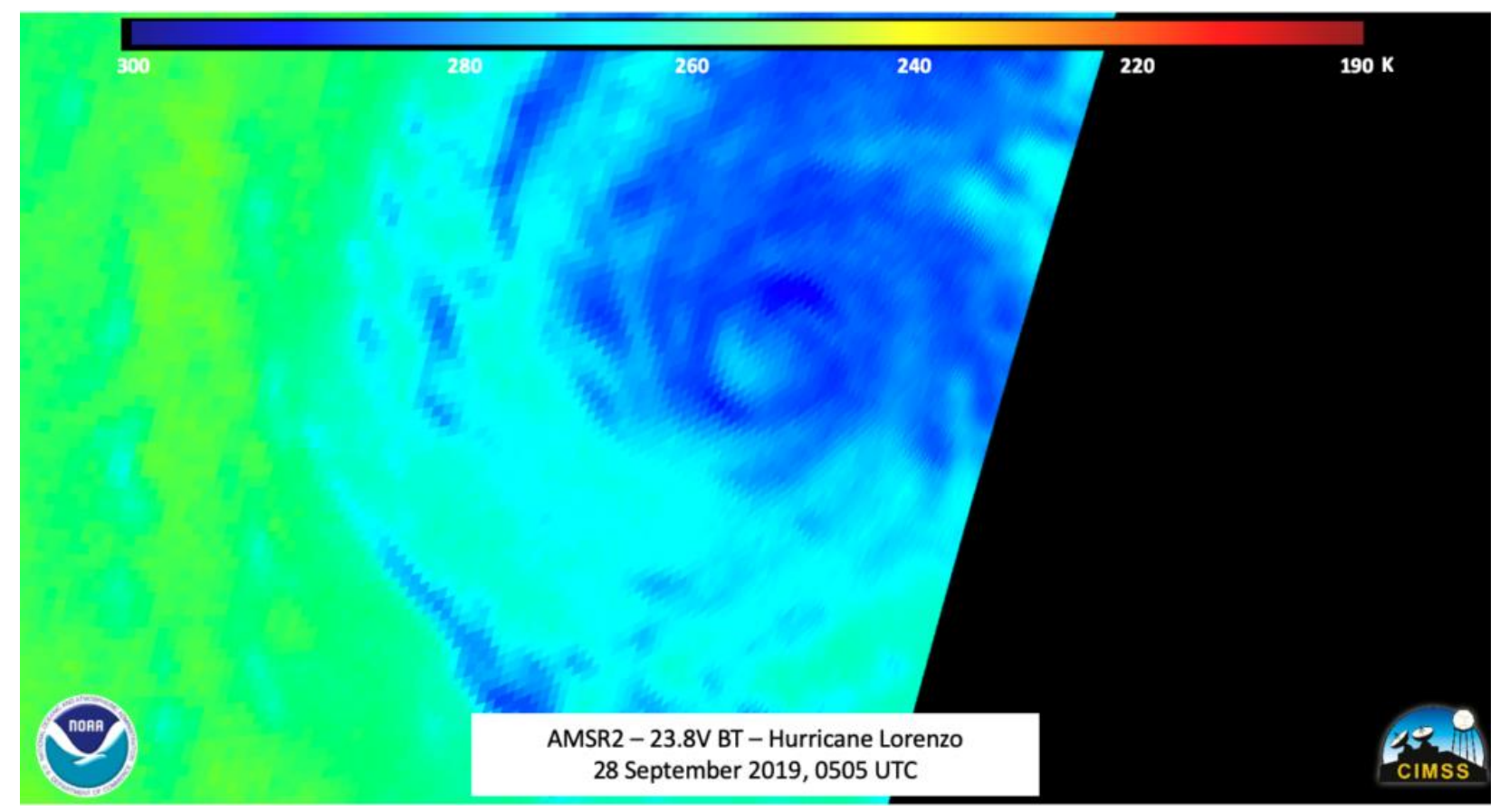

Figure 2. Microwave sensed image from the Advanced Microwave Scanning Radiometer 2 (AMSR2) of Hurricane Lorenzo from September 28, 2019. This image utilizes parts of the spectra proposed for $5 \mathrm{G}$ and allows a view of the hurricane from different levels of the atmosphere. When the eye of a hurricane is obscured by clouds, microwave imagery like AMSR2 is used to capture the eye location, providing valuable information about the storm necessary for ensuring the safety of life and property. Images used by permission from William Straka/CIMSS.

\section{References}

Allianz Global Corporate \& Specialty. 2013. “Superstorm Sandy: Lessons Learned: A risk management perspective."

https://www.allianz.com/content/dam/onemarket ing/azcom/Allianz_com/migration/media/press/d ocument/other/Sandy risk bulletin.pdf

Bauer, Peter, Alan Thorpe, and Gilbert Brunet. 2015. "The Quiet Revolution of Numerical Weather Prediction." Nature 525 (7567): 47-55. https://doi.org/10.1038/nature14956.

Bormann, Niels, Heather Lawrence, and Jacky Farnan. 2019. "Global Observing System Experiments in the ECMWF Assimilation System." Technical Memorandum 839. ECMWF Technical Memoranda. European Centre for Medium-Range Weather Forecasts (ECMWF). https://pdfs.semanticscholar.org/8fd5/146b5f6ef3 d0530eb2d360363ad4c7d36d64.pdf.

Campbell, Karen, Liz Cruz, Bob Flanagan, Bill Morelli, Brendan O’Neil, Stéphane Téral, and Julian Watson.
2019. "The 5G Economy: How 5G Will Contribute to the Global Economy." IHS Markit.

https://www.qualcomm.com/media/documents/fi les/ihs-5g-economic-impact-study-2019.pdf.

Commercial Spectrum Enhancement Act, U.S. Code 47 (2003), §§ 901 et seq.

901.https://legcounsel.house.gov/Comps/Publi\%2 0Law\%20108-494.pdf.

Dutton, John A. 2002. "Opportunities and Priorities in a New Era for Weather and Climate Services." Bulletin of the American Meteorological Society 83 (9): 1303-12. https://doi.org/10.1175/1520-047783.9.1303.

ECMWF (European Centre for Medium-Range Weather Forecasts). 2018. "Radio-Frequency Interference (RFI) Workshop." Shinfield Park, Reading, Berkshire, RG2 9AX, UK. https://www.ecmwf.int/sites/default/files/elibrar y/2019/19026-radio-frequency-interference-rfiworkshop-final-report.pdf. 
FCC (Federal Communications Commission). 2016. "The FCC's 5G FAST Plan.” FCC Initiatives. September 15, 2016. https://www.fcc.gov/5G.

FCC (Federal Communications Commission). 2020. "Radio Spectrum Allocation.” Federal Communications Commission. March 2020. https://www.fcc.gov/engineeringtechnology/policy-and-rulesdivision/general/radio-spectrum-allocation.

Freedman, Andrew. 2019. "Weather Is Turning into Big Business. And That Could Be Trouble for the Public." Washington Post, November 25, 2019. https://www.washingtonpost.com/business/2019 /11/25/weather-is-big-business-its-veeringtoward-collision-with-federal-government/.

Greensill. 2019. "Financing the Future of 5G." https://greensillwebsite.s3.amazonaws.com/uploa ds/2019/10/Greensill 5G oct 2019.pdf

JPSS Satellite-based Disaster Outreach Coordinator (SDOC). "Hurricane Lorenzo from morning of 28 September 2019." Cooperative Institute for Meteorological Satellite Studies (CIMSS). https://www.ssec.wisc.edu/jpsssdoc/posts/hurricane-lorenzo/hurricane-lorenzofrom-morning-of-28-september-2019/.

Lazo, Jeffrey K., Megan Lawson, Peter H. Larsen, and Donald M. Waldman. 2011. "U.S. Economic Sensitivity to Weather Variability." Bulletin of the American Meteorological Society 92 (6): 709-20. https://doi.org/10.1175/2011BAMS2928.1.

Lubar, David G., David B. Kunkee, Lina M. Cashin, and Susan K. Avery. 2020. "Developing a Sustainable Spectrum Approach to Deliver 5G Services and Critical Weather Forecasts." The Aerospace Corporation - Center for Space Policy and Strategy. https://aerospace.org/sites/default/files/202001/LubarKunkee DevelopingSustSpectrum 20200 109 web.pdf.

McNally, Tony, Massimo Bonavita, and Jean-Noël Thépaut. 2014. "The Role of Satellite Data in the Forecasting of Hurricane Sandy." Monthly Weather Review 142 (2): 634-46. https://doi.org/10.1175/MWR-D-13$\underline{00170.1}$.

Miao, Guowang, Jens Zander, Ki Won Sung, and Silmane Ben Silmane. "Fundamentals of Mobile Data Networks." Cambridge University Press, 2016. https://www.google.com/books/edition/Fundame ntals of Mobile Data Networks/ImeSCwAAQBAJ?h $\underline{l=e n \& g b p v=0}$.

NASA (National Aeronautics and Space Administration). 2020. "GEOS Observation Impact Monitoring." Global Modeling and Assimilation Office. 2020. https://gmao.gsfc.nasa.gov/forecasts/systems/fp/ obs impact/.

NASA (National Aeronautics and Space Administration), and NOAA (National Oceanic and Atmospheric Administration). 2020. "NASA/NOAA Sharing
Studies on WRC-19 Agenda Item 1.13.Pdf."

https://science.house.gov/imo/media/doc/Study

$\% 20$ prepared $\% 20$ by $\% 20$ NOAA $\% 20$ and $\% 20$ NASA

$\% 20-$

$\% 20$ Results $\% 20$ from $\% 20$ NASANOAA $\% 20$ Sharing

\%20Studies\%20on\%20WRC-

19\%20Agenda\%20Item\%201.13.pdf.

National Integrated Drought Information System

Reauthorization Act of 2018, U.S. Code 15 (2019), §§

$313 d$ et seq.

https://www.congress.gov/115/plaws/publ423/P

LAW-115publ423.pdf

NOAA (National Oceanic and Atmospheric

Administration) National Environmental Satellite,

Data, and Information Service (NESDIS). 2019. “A

Guide to Understanding Satellite Images of

Hurricanes"

https://www.nesdis.noaa.gov/content/guideunderstanding-satellite-images-hurricanes

NOAA (National Oceanic and Atmospheric Administration) NCEI (National Centers For Environmental Information). 2020. "U.S. BillionDollar Weather and Climate Disasters, 1980 Present (NCEI Accession 0209268)." NOAA National Centers for Environmental Information. https://doi.org/10.25921/STKW-7W73.

NWS (National Weather Service). 2017. "National Weather Service Enterprise Analysis Report: Findings on Changes in the Private Weather Industry."

https://www.weather.gov/media/about/Final_NW S\%20Enterprise\%20Analysis $\% 20$ Report June $\% 20$ 2017.pdf.

US Congress. House. Committee on Science, Space \& Technology. The Future of Forecasting: Building a Stronger U.S. Weather Enterprise: Hearing before the Subcommittee on the Environment, 116th Cong., 1st session., May 16, 2019.

https://science.house.gov/hearings/the-future-offorecasting-building-a-stronger-us-weatherenterprise

US Congress. House. Studying How to Harness Airwave Resources Efficiently (SHARE Act) Act of 2019. HR 5000. 116th Cong., 1st sess. Introduced in House November 8, 2019.

https://www.congress.gov/116/bills/hr5000/BILL S-116hr5000ih.pdf.

Weather Research and Forecasting Act of 2017, U.S. Code 15 (2017), §§ 8501 et seq. https://www.congress.gov/115/plaws/publ25/PL AW-115publ25.pdf

White House. 2018. "National Cyber Strategy of the United States of America."

https://www.whitehouse.gov/wpcontent/uploads/2018/09/National-CyberStrategy.pdf. 
White House. 2020. "National Strategy to Secure 5G of the United States of America."

https://www.whitehouse.gov/wpcontent/uploads/2020/03/National-Strategy-5GFinal.pdf.
White House OMB (Office of Management and Budget). 2020. "Budget of the U.S. Government for Fiscal

Year 2021." ISBN 978-0-16-095401-6. https://www.whitehouse.gov/wpcontent/uploads/2020/02/budget fy21.pdf.

World Economic Forum. 2020. "The Impact of 5G: Creating New Value across Industries and Society." World Economic Forum.

http://www3.weforum.org/docs/WEF The Impact of 5G Report.pdf

Sarah Benish is a Ph.D. candidate at the University of Maryland in the Department of Atmospheric and Oceanic Science studying air pollutants and greenhouse gases from an airborne field campaign in the North China Plain. She is a founding member and Vice-President of Graduate Science Policy at the University of Maryland. Sarah earned a BS in Biology and Environmental Studies with a certificate in Global Health from the University of Wisconsin-Madison.

Rachel Lamb is a Ph.D. candidate in the Department of Geographical Sciences at the University of Maryland, College Park (UMD). In partnership with NASA's Carbon Monitoring System, Rachel is working with state governments to better include land-based carbon in their climate change mitigation planning and activities. She has served in multiple leadership capacities on campus including as the Vice President of Government Affairs for Graduate Student Government, and founding member and treasurer of Graduate Science Policy at UMD. Rachel also earned Masters degrees in Public Policy, and in Sustainable Development and Conservation Biology from UMD, as well as a BS in Environmental Studies and BA in International Relations from Wheaton College (IL).

Abhinav Deshpande is a Ph.D. candidate at the University of Maryland in the Department of Physics. His research interests are at the intersection of quantum computer science and many-body physics. He was previously at the Indian Institute of Technology, Kanpur, where he obtained an integrated bachelor's and master's degree in physics.

Graham Reid is a Ph.D. candidate in the Physics Department at the University of Maryland where he works in atomic physics experiment. His research uses ultracold atoms as a model to study transport in disordered quantum systems.

Shantam Ravan is a Ph.D candidate in the Physics Department at the University of Maryland. He currently works on studying nitrogen vacancies in diamond for use in high-resolution nanoscale sensing, and as a model system for driven many-body interacting quantum systems. He earned a BS in Electrical Engineering and Engineering Physics from the University of Michigan.

\section{Acknowledgements}

The authors would like to thank Dr. Adria Schwarber and Dr. Yuhan (Douglas) Rao for their review of and comments on our manuscript. We would also like to thank Aditi Dubey and Liz Friedman, executive members of Graduate Science Policy at University of Maryland, for their feedback on earlier drafts. 